\title{
$\begin{array}{ll}\text { Research Square } & \begin{array}{l}\text { Preprints are preliminary reports that have not undergone peer review. } \\ \text { They should not be considered conclusive, used to inform clinical practice, } \\ \text { or referenced by the media as validated information. }\end{array}\end{array}$
}

\section{The Efficacy and Safety of Tranexamic Acid Combined with Rivaroxaban in Prevention of Clinical Events in Patients after Total Knee/ Hip Arthroplasty: A Meta-analysis}

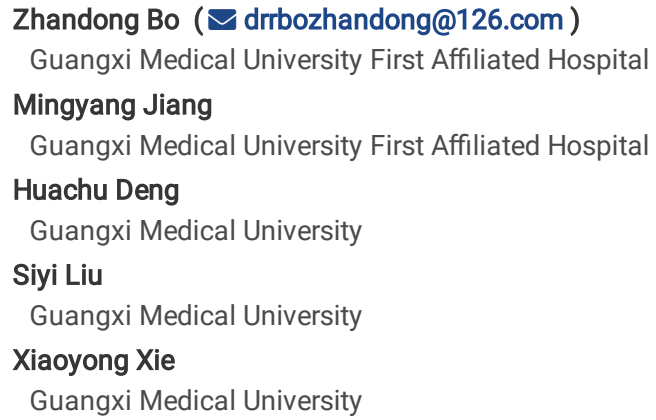




\section{Abstract}

Purpose

To evaluate the efficacy and safety of tranexamic acid combined with rivaroxaban in prevention of clinical events in patients after TKA/THA through metaanalysis of randomized controlled trials.

Materials and Methods

RCTs were retrieved from medical literature databases. RR, SMD and 95\% confidence intervals $(\mathrm{Cl})$ were calculated to compare the primary and safety endpoints.

Results

In total, 16 articles (23 trial comparisons) were retrieved which contained 2179 patients. In general, 1257 patients (57.7\%) were randomized to experimental group whereas 922 patients (42.3\%) were randomized to control group. The result showed that TXA combined with rivaroxaban significantly reduce TBL, BTV, BTR and the incidence of MB compared to the control group; there were no significant differences in NMB between experimental group and control group.

\section{Conclusions}

This meta-analysis reveals that TXA combined with rivaroxaban can significantly reduce TBL, BTV, the incidence of blood transfusion and the incidence of MB compared to the control group, which proved that its efficacy and safety are trustworthy.

\section{Background}

In recent years, total knee arthroplasty (TKA) has become an important method for the clinical treatment of severe knee joint diseases, while total hip arthroplasty (THA) is also widely used in the treatment of end-stage femoral head necrosis, hip ankylosis and other hip related diseases [1]. As the techniques of TKA and THA become more and more mature, one of the biggest problems that afflict these two types of surgery are the large amount of blood loss during the perioperative period and the need for blood transfusion after operation [2]. Therefore, the prevention and treatment of perioperative complications and postoperative rehabilitation on the success or failure of the operation and to ensure the postoperative recovery of patients cannot be ignored [3].

TXA is a commonly used hemostatic drug in clinic, which can competitively prevent and inhibit the binding of fibrin with fibrinogen and fibrinolytic enzyme, and then play a hemostatic effect, several studies have confirmed that the use of TXA before and during THA/TKA can effectively reduce blood loss [4]. However, there may be a risk of keeping venous blood in a hypercoagulable state at the same time $[5,6]$. Patients undergoing major orthopedic surgery, especially lower limb joint replacement, are inherently at high risk of venous thromboembolism (VTE). Both the American Academy of Orthopedic Surgeons (AAOS) and the American College of Chest Physicians (ACCP) have developed new evidence-based guidelines for venous thromboembolic prophylaxis after total joint arthroplasty $[7,8]$.

Low molecular weight heparin (LMWH) is still commonly used in anticoagulant, but LMWH needs to be adjusted when it is used, and subcutaneous injection leads to poor compliance of patients after discharge. Rivaroxaban is a direct oral anticoagulant, which is used to prevent VTE in THA/TKA [9]. At the same time, rivaroxaban is given orally without adjusting the dose. Despite its clinical efficacy in VTE prophylaxis, orthopedic surgeons are still skeptical regarding the routine use of rivaroxaban in knee and hip surgery and, in particular, the increased risk of bleeding complications [10].

Therefore, how to balance antifibrinolysis and anticoagulation is a challenge. Some studies have pointed out that we should guard against the risk of postoperative VTE associated with antifibrinolytic drugs and the risk of bleeding caused by anticoagulant drugs [5, 6]. There are also studies suggest that TXA has a short half-life in plasma and its antifibrinolytic effect only lasts for 3-4 hours [11], or according to others, up to 6-8 hours [12]. This time interval is well shorter than, or may just coincide with, the initiation of anticoagulant administration to patients after joint replacement surgery. In theory, therefore, no "contradiction" in the combined use of such agents exists, the added use of TXA does not increase rates of thromboembolic events after total joint replacement surgery [13-15]. The application of TXA combined with rivaroxaban is relatively few and lacks clinical significance. The purpose of this study was to explore the efficacy and safety of TXA combined with rivaroxaban in patients with THA or TKA to provide more options for the clinical application of anticoagulants.

\section{Methods Search strategy}

Two researchers searched for published articles comparing the efficacy and safety of TXA combined with rivaroxaban in patients with THA or TKA following the Preferred Reporting Items for Systematic Reviews and Meta-Analyses (PRISMA) guidelines. The RCTs were systematically searched in the databases such as the Cochrane Library, Embase, PubMed, Google Scholar, Baidu Scholar, CNKI and VIP with no restrictions on language or publication date from inception to 16 May 2019. The following keywords and MeSH terms were used: ("total knee arthroplasty" OR "total hip arthroplasty") AND "tranexamic acid" AND "rivaroxaban". Additional relevant studies were retrieved from reviews, meta-analyses, and other literature. Two authors screened and double-reviewed the retrieved studies. Where disputes were encountered, they were resolved by consulting a third author. In this meta-analysis, all data were extracted from previously published studies, thus no patient consent and ethical approval were required. 


\section{Inclusion And Exclusion Criteria}

The following inclusion criteria were used: (1) Studies that assessed the efficacy and safety of TXA combined with rivaroxaban in patients with THA or TKA; (2) The study was a randomized controlled trial (RCT); (3) The study subjects were patients undergoing THA or TKA (both primary and revision cases); (4) General information (e.g. gender, age, disease type) of the experimental group and the control group was not statistically different at baseline; (5) At least one of the evaluated groups was based on TXA combined with rivaroxaban; (6) TXA and rivaroxaban had no limitation in usage and dose; (7) Included articles provide sufficient data for analysis; (8) Language was limited to English or Chinese.

The following exclusion criteria were used: (1) Nonclinical trials, case reports or series; (2) Animal experiments; (3) Semi-randomized controlled trials or nonrandomized trials; (4) Articles with incorrect or incomplete data, or articles whose data could not be extracted; (5) Studies that compared the efficacy and safety of TXA versus rivaroxaban in patients after THA or TKA.

\section{Endpoints}

The primary endpoints for this study were total blood loss (TBL), blood transfusion volume (BTV) and blood transfusion rate (BTR). The secondary endpoints for this study were hidden blood loss (HBL), intraoperative blood loss (IBL), postoperative drainage, (activated partial thromboplastin time) APTT, (fibrinogen) FG, (hemoglobin) Hb and (prothrombin time) PT. The safety endpoints included major bleeding (MB), non-major bleeding (NMB) (including clinically relevant non-major bleeding, minor bleeding, any overt bleeding, etc) and venous thromboembolism (VTE).

\section{Data Extraction}

Two authors independently reviewed the contents of the retrieved studies. The primary endpoints were extracted by two authors and verified by a third author. The data extracted included the following primary information: first author's name, year of publication, test type/region, sample size, sex ratio, average age, body mass index (BMI), intervention, operative type, follow-up time and endpoints measured in each study. If the contents of the studies needed clarification, the first author of the study was contacted. Disagreements were resolved through consensus or by consulting a third author.

\section{Risk-of-bias Assessments}

The methodological quality of the included studies was estimated independently by two authors based on The Cochrane Risk of Bias criteria. Each quality item was graded as low risk, high risk, or no clear risk. The seven items used to assess bias in each trial included the randomization sequence generation, allocation concealment, blinding of participants and personnel, blinding of outcome assessment, incomplete outcome data, selective reporting, and other biases.

\section{Statistical analysis}

Stata (version 12.0, Stata Corp, College Station, Texas) was used to analyze and pool the individual research results. Pooled results were recorded as risk ratios (RR) Standard mean difference (SMD) and 95\% confidence intervals (CI) with two-sided P-values. P-values $<0.05$ were considered to be statistically significant. Heterogeneity was evaluated using the $\mathrm{I}^{2}$ test. The heterogeneity was considered to be small when $\mathrm{I}^{2}<50 \%$ and substantial when $\mathrm{I}^{2}>50 \%$. The fixed effect model was used when $\mathrm{I}^{2}<50 \%$, while the random effect model was used when $\mathrm{I}^{2}>50 \%$. A funnel plot was generated to examine the publication bias and to explore the sources of heterogeneity if more than ten studies were included to assess this endpoint. Subgroup analysis was performed according to the administration, operative type, follow-up period and dosage of TXA.

\section{Results}

\section{Studies Retrieved and Characteristics}

A total of 3298 relevant studies were enrolled according to PRISMA guidelines. The titles and abstracts of the studies were screened to exclude irrelevant studies. Then, we further eliminated the unfit studies by reading the full text of the articles. Finally, 16 studies [12, 16-30] (23 trial comparisons) were included according to the inclusion and exclusion criteria and they had a total of 2179 patients as shown in Fig. 1. In general, 1257 patients (57.7\%) were randomized to experimental group whereas 922 patients (42.3\%) were randomized to control group. All studies included in this meta-analysis were RCTs. The basic characteristics of the individuals from the trials are described in Table 1. 
Table 1

Characteristics of studies included in meta-analysis.

\begin{tabular}{|c|c|c|c|c|c|c|c|c|c|c|c|c|c|}
\hline \multirow[t]{2}{*}{ Author } & \multirow[t]{2}{*}{ Year } & \multirow[t]{2}{*}{ Country } & \multicolumn{2}{|c|}{ Sample size } & \multicolumn{2}{|c|}{ Women, No. (\%) } & \multicolumn{2}{|c|}{$\begin{array}{l}\text { Average age } \\
\text { (years) }\end{array}$} & \multicolumn{2}{|l|}{ BMI } & \multicolumn{2}{|l|}{ Intervention } & \multirow[t]{2}{*}{$\begin{array}{l}\text { Op } \\
\text { typ }\end{array}$} \\
\hline & & & E & C & $E$ & C & $E$ & C & $E$ & C & $E$ & C & \\
\hline $\begin{array}{l}\text { Jianbao } \\
\text { Li[16] }\end{array}$ & 2014 & China & 45 & 45 & $25(55.5)$ & $21(46.6)$ & $\begin{array}{l}55.47 \\
\pm \\
13.32\end{array}$ & $\begin{array}{l}52.58 \\
\pm \\
14.21\end{array}$ & N/A & N/A & $\begin{array}{l}\text { TXA: } 15 \mathrm{mg} / \mathrm{kg} \text {, tid, } \\
\text { intravenous drip. } \\
\text { Rivoraxaban: } \\
10 \text { mg/day, orally. }\end{array}$ & $\begin{array}{l}\text { Rivoraxaban: } \\
10 \mathrm{mg} / \text { day, } \\
\text { orally. }\end{array}$ & Tr \\
\hline $\begin{array}{l}\text { Guokuan } \\
\text { Xing[17] }\end{array}$ & 2015 & China & 60 & 60 & $42(70.0)$ & $46(76.6)$ & $\begin{array}{l}70.34 \\
\pm 7.41\end{array}$ & $\begin{array}{l}69.32 \\
\pm 7.21\end{array}$ & 27.3 & 26.7 & $\begin{array}{l}\text { TXA: } 1.5 \mathrm{~g} \text {, intraarticular } \\
\text { injection; } 1.0 \mathrm{~g} \text { local } \\
\text { perfusion. } \\
\text { Rivoraxaban: } \\
15 \mathrm{mg} / \text { day, orally. }\end{array}$ & $\begin{array}{l}\text { LMWH: } 2500 \\
\text { AXa IU /d, } \\
\text { hypodermic } \\
\text { injection. }\end{array}$ & TK \\
\hline $\begin{array}{l}\text { Junwen } \\
\text { Wang[18] }\end{array}$ & 2016 & China & 100 & 98 & $74(74.0)$ & 72(77.6) & 68.19 & 69.60 & 28.2 & 28.1 & $\begin{array}{l}\text { TXA: } 1.0 \mathrm{~g} \text {, } \\
\text { intraoperative injection. } \\
\text { Rivoraxaban: } \\
10 \mathrm{mg} / \text { day, orally. }\end{array}$ & $\begin{array}{l}\text { Rivoraxaban: } \\
10 \mathrm{mg} / \text { day, } \\
\text { orally. }\end{array}$ & TK \\
\hline $\begin{array}{l}\text { Fulin Li } \\
\text { a[19] }\end{array}$ & 2017 & China & 30 & 30 & $7(23.3)$ & 10(33.3) & $\begin{array}{l}62.20 \\
\pm 6.60\end{array}$ & $\begin{array}{l}62.20 \\
\pm 7.30\end{array}$ & 21.8 & 21.3 & $\begin{array}{l}\text { TXA: } 10 \mathrm{mg} / \mathrm{kg} \text { before } \\
\text { skin incision, } \\
\text { intravenous drip. } \\
\text { Rivoraxaban: } \\
10 \mathrm{mg} / \text { day, orally. }\end{array}$ & $\begin{array}{l}100 \mathrm{ml} 0.9 \% \\
\mathrm{NS} \text {, } \\
\text { intravenous } \\
\text { drip }\end{array}$ & Tr \\
\hline $\begin{array}{l}\text { Fulin Li } \\
\text { b[19] }\end{array}$ & 2017 & China & 30 & 30 & $8(26.7)$ & 10(33.3) & $\begin{array}{l}62.70 \\
\pm 6.50\end{array}$ & $\begin{array}{l}62.20 \\
\pm 7.30\end{array}$ & 21.4 & 21.3 & $\begin{array}{l}\text { TXA: } 15 \mathrm{mg} / \mathrm{kg} \text {, before } \\
\text { skin incision, } \\
\text { intravenous drip. } \\
\text { Rivoraxaban: } \\
10 \mathrm{mg} / \text { day, orally. }\end{array}$ & $\begin{array}{l}100 \mathrm{ml} 0.9 \% \\
\text { NS: } \\
\text { intravenous } \\
\text { drip. }\end{array}$ & Tr \\
\hline $\begin{array}{l}\text { Fulin Li } \\
\text { c[19] }\end{array}$ & 2017 & China & 30 & 30 & $9(30.0)$ & 10(33.3) & $\begin{array}{l}61.80 \\
\pm \\
13.00\end{array}$ & $\begin{array}{l}62.20 \\
\pm 7.30\end{array}$ & 21.1 & 21.3 & $\begin{array}{l}\text { TXA: } 15 \mathrm{mg} / \mathrm{kg} \text {, before } \\
\text { skin incision and after } \\
3 \mathrm{~h} \text {, intravenous drip. } \\
\text { Rivoraxaban: } \\
10 \mathrm{mg} / \text { day, orally. }\end{array}$ & $\begin{array}{l}100 \mathrm{ml} 0.9 \% \\
\text { NS: } \\
\text { intravenous } \\
\text { drip. }\end{array}$ & Tr \\
\hline $\begin{array}{l}\text { Fulin Li } \\
\text { d[19] }\end{array}$ & 2017 & China & 30 & 30 & $8(26.7)$ & 10(33.3) & $\begin{array}{l}62.40 \\
\pm 7.40\end{array}$ & $\begin{array}{l}62.20 \\
\pm 7.30\end{array}$ & 21.4 & 21.3 & $\begin{array}{l}\text { TXA: } 15 \mathrm{mg} / \mathrm{kg} \text {, before } \\
\text { skin incision, } \\
\text { intravenous drip; } 1.0 \mathrm{~g} \text {, } \\
\text { local wet compress. } \\
\text { Rivoraxaban: } \\
10 \mathrm{mg} / \text { day, orally. }\end{array}$ & $\begin{array}{l}100 \mathrm{ml} 0.9 \% \\
\text { NS: } \\
\text { intravenous } \\
\text { drip. }\end{array}$ & Tr \\
\hline $\begin{array}{l}\text { Guokuan } \\
\text { Xing[20] }\end{array}$ & 2017 & China & 50 & 50 & $36(72.0)$ & $38(76.0)$ & $\begin{array}{l}61.40 \\
\pm 6.60\end{array}$ & $\begin{array}{l}63.10 \\
\pm 3.80\end{array}$ & 26.3 & 27.4 & $\begin{array}{l}\text { TXA: } 1.0 \mathrm{~g} \text {, intravenous } \\
\text { drip; } 1.0 \mathrm{~g} \text {, local } \\
\text { perfusion. } \\
\text { Rivoraxaban: } \\
15 \mathrm{mg} / \text { day, orally. }\end{array}$ & $\begin{array}{l}\text { LMWH: } 2500 \\
\text { AXa IU /d, } \\
\text { hypodermic } \\
\text { injection. }\end{array}$ & TK \\
\hline $\begin{array}{l}\text { Hongjian } \\
\text { Xu[21] }\end{array}$ & 2017 & China & 75 & 75 & $35(46.6)$ & $34(45.3)$ & $\begin{array}{l}65.18 \\
\pm 3.32\end{array}$ & $\begin{array}{l}65.22 \\
\pm 3.28\end{array}$ & N/A & N/A & $\begin{array}{l}\text { TXA: } 1.0 \mathrm{~g} \text {, local wet } \\
\text { compress. } \\
\text { Rivoraxaban: } \\
10 \mathrm{mg} / \text { day, orally. }\end{array}$ & $\begin{array}{l}\text { LMWH: } \\
0.01 \mathrm{ml} / \mathrm{kg} \text {, } \\
\text { q12h, } \\
\text { hypodermic } \\
\text { injection. }\end{array}$ & Tr \\
\hline $\begin{array}{l}\text { Jinwei } \\
\text { Xie[22] }\end{array}$ & 2017 & China & 96 & 98 & $74(96.0)$ & $86(98.0)$ & $\begin{array}{l}65.20 \\
\pm 5.50\end{array}$ & $\begin{array}{l}66.80 \\
\pm 7.40\end{array}$ & 25.4 & 25.6 & $\begin{array}{l}\text { TXA: } 15 \mathrm{mg} / \mathrm{kg} \text {, } \\
\text { intravenous before } \\
\text { tourniquet deflation; } \\
1.0 \mathrm{~g} \text {, injected into the } \\
\text { articular cavity through } \\
\text { the drainage tube. } \\
\text { Rivoraxaban: } \\
10 \mathrm{mg} / \text { day, orally. }\end{array}$ & $\begin{array}{l}\text { TXA: } \\
15 \mathrm{mg} / \mathrm{kg}, \\
\text { intravenous } \\
\text { before } \\
\text { tourniquet } \\
\text { deflation; } \\
1.0 \mathrm{~g} \text {, injected } \\
\text { into the } \\
\text { articular } \\
\text { cavity through } \\
\text { the drainage } \\
\text { tube. } \\
\text { LMWH: } 0.4 \mathrm{ml} \\
4000 \text { IU, } \\
\text { hypodermic } \\
\text { injection. }\end{array}$ & TK \\
\hline $\begin{array}{l}\text { Keming } \\
\text { Xia a[23] }\end{array}$ & 2017 & China & 49 & 49 & N/A & $\mathrm{N} / \mathrm{A}$ & $\begin{array}{l}66.00 \\
\pm \\
10.40\end{array}$ & $\begin{array}{l}62.40 \\
\pm \\
11.30\end{array}$ & $\begin{array}{l}25.4 \\
\pm 3.9\end{array}$ & $\begin{array}{l}25.4 \\
\pm 3.3\end{array}$ & $\begin{array}{l}\text { TXA: } 10 \mathrm{mg} / \mathrm{kg}, 0.5 \mathrm{~h} \\
\text { before operation, } \\
\text { intravenous. } \\
\text { Rivoraxaban: } \\
10 \mathrm{mg} / \text { day, orally. }\end{array}$ & $\begin{array}{l}\text { Rivoraxaban: } \\
10 \mathrm{mg} / \text { day, } \\
\text { orally. }\end{array}$ & TK \\
\hline
\end{tabular}




\begin{tabular}{|c|c|c|c|c|c|c|c|c|c|c|c|c|c|}
\hline \multirow[t]{2}{*}{ Author } & \multirow[t]{2}{*}{ Year } & \multirow[t]{2}{*}{ Country } & \multicolumn{2}{|c|}{ Sample size } & \multicolumn{2}{|c|}{ Women, No. (\%) } & \multicolumn{2}{|c|}{$\begin{array}{l}\text { Average age } \\
\text { (years) }\end{array}$} & \multicolumn{2}{|l|}{ BMI } & \multicolumn{2}{|l|}{ Intervention } & \multirow[t]{2}{*}{$\begin{array}{l}\text { Op } \\
\text { typ }\end{array}$} \\
\hline & & & $E$ & C & $E$ & C & $E$ & C & $E$ & $\mathrm{C}$ & $E$ & C & \\
\hline $\begin{array}{l}\text { Keming } \\
\text { Xia b[23] }\end{array}$ & 2017 & China & 49 & 49 & $\mathrm{~N} / \mathrm{A}$ & $\mathrm{N} / \mathrm{A}$ & $\begin{array}{l}64.10 \\
\pm 9.20\end{array}$ & $\begin{array}{l}62.40 \\
\pm \\
11.30\end{array}$ & $\begin{array}{l}25.9 \\
\pm 4.1\end{array}$ & $\begin{array}{l}25.4 \\
\pm 3.3\end{array}$ & $\begin{array}{l}\text { TXA: } 10 \mathrm{mg} / \mathrm{kg}, 0.5 \mathrm{~h} \\
\text { before operation, } \\
\text { intravenous; } 50 \mathrm{ml} \text {, local } \\
\text { injection after operation. } \\
\text { Rivoraxaban: } \\
10 \mathrm{mg} / \text { day, orally. }\end{array}$ & $\begin{array}{l}\text { Rivoraxaban: } \\
10 \mathrm{mg} / \text { day, } \\
\text { orally. }\end{array}$ & TK \\
\hline $\begin{array}{l}\text { Shih- } \\
\text { Hsiang } \\
\text { Yen a[24] }\end{array}$ & 2017 & China & 31 & 30 & 27(87.1) & $24(80.0)$ & 69.13 & 70.87 & 28.4 & 28.3 & $\begin{array}{l}\text { TXA: } 1.0 \mathrm{~g} \text {, } \\
\text { intraoperative bolus } \\
\text { injection. } \\
\text { Rivoraxaban: } \\
10 \text { mg/day, orally. }\end{array}$ & $\begin{array}{l}\text { Rivoraxaban: } \\
10 \mathrm{mg} / \text { day, } \\
\text { orally. }\end{array}$ & TK \\
\hline $\begin{array}{l}\text { Shih- } \\
\text { Hsiang } \\
\text { Yen b[24] }\end{array}$ & 2017 & China & 32 & 30 & 19(59.4) & $24(80.0)$ & 69.66 & 70.87 & 28.1 & 28.3 & $\begin{array}{l}\text { TXA: } 3.0 \mathrm{~g} \text {, intraarticular } \\
\text { injection. } \\
\text { Rivoraxaban: } 10 \mathrm{mg} / \mathrm{day} \text {, } \\
\text { orally. }\end{array}$ & $\begin{array}{l}\text { Rivoraxaban: } \\
10 \mathrm{mg} / \text { day, } \\
\text { orally. }\end{array}$ & TK \\
\hline $\begin{array}{l}\text { Weina } \\
\text { Zhou[25] }\end{array}$ & 2017 & China & 33 & 33 & $\mathrm{~N} / \mathrm{A}$ & $\mathrm{N} / \mathrm{A}$ & $\begin{array}{l}66.30 \\
\pm 7.40\end{array}$ & $\begin{array}{l}66.30 \\
\pm 7.40\end{array}$ & 23.9 & 23.9 & $\begin{array}{l}\text { TXA: } 1.0 \mathrm{~g} \text {, intraarticular } \\
\text { injection. } \\
\text { Rivoraxaban: } 15 \mathrm{mg} / \mathrm{day} \text {, } \\
\text { orally. }\end{array}$ & $\begin{array}{l}\text { TXA: } 1.0 \mathrm{~g} \\
\text { intraarticular } \\
\text { injection. } \\
\text { LMWH: } \\
2500 \text { IU /d, } \\
\text { hypodermic } \\
\text { injection. }\end{array}$ & Tr \\
\hline $\begin{array}{l}\text { Yanmei } \\
\text { Fan[26] }\end{array}$ & 2017 & China & 65 & 65 & $\mathrm{~N} / \mathrm{A}$ & $\mathrm{N} / \mathrm{A}$ & $\begin{array}{l}60 \sim \\
87\end{array}$ & $\begin{array}{l}60 \sim \\
87\end{array}$ & N/A & N/A & $\begin{array}{l}\text { TXA: } 0.5 \mathrm{~g} \text {, local wet } \\
\text { compress. } \\
\text { Rivoraxaban: } \\
10 \mathrm{mg} / \text { day, orally. }\end{array}$ & $\begin{array}{l}\text { Rivoraxaban: } \\
10 \mathrm{mg} / \text { day, } \\
\text { orally. }\end{array}$ & TK \\
\hline $\begin{array}{l}\text { Yanan } \\
\text { Fan[27] }\end{array}$ & 2017 & China & 30 & 30 & 13(43.3) & 10(33.3) & $\begin{array}{l}57.03 \\
\pm \\
10.23\end{array}$ & $\begin{array}{l}55.55 \\
\pm 7.11\end{array}$ & $\begin{array}{l}22.8 \\
\pm 2.7\end{array}$ & $\begin{array}{l}23.4 \\
\pm 3.5\end{array}$ & $\begin{array}{l}\text { TXA: } 1.0 \text { g, preoperative } \\
\text { intravenous drip; } 100 \mathrm{ml} \text {, } \\
\text { after sewing. } \\
\text { Rivoraxaban: } \\
10 \text { mg/day, orally. }\end{array}$ & $\begin{array}{l}\text { TXA: } 1.0 \mathrm{~g} \text {, } \\
\text { preoperative } \\
\text { intravenous } \\
\text { drip; } 100 \mathrm{ml} \text {, } \\
\text { after sewing. } \\
\text { LMWH: drug } \\
\text { administration } \\
\text { as appropriate }\end{array}$ & Tr \\
\hline $\begin{array}{l}\text { Fang Lan } \\
\text { a[28] }\end{array}$ & 2018 & China & 70 & 78 & $37(52.9)$ & $46(58.9)$ & $\begin{array}{l}62.50 \\
\pm \\
15.40\end{array}$ & $\begin{array}{l}63.70 \\
\pm \\
11.90\end{array}$ & N/A & N/A & $\begin{array}{l}\text { TXA: } 10 \mathrm{mg} / \mathrm{kg} \\
\text { (preoperative } \\
\text { intravenous drip) } \\
10 \mathrm{mg} / \mathrm{kg} \\
\text { (postoperative } \\
\text { intravenous drip) } \\
\text { Rivoraxaban: } \\
10 \mathrm{mg} / \text { day, orally. }\end{array}$ & $\begin{array}{l}250 \mathrm{ml} 0.9 \% \\
\text { NS: } \\
\text { intravenous } \\
\text { drip. }\end{array}$ & TK \\
\hline $\begin{array}{l}\text { Fang Lan } \\
\text { b[28] }\end{array}$ & 2018 & China & 92 & 78 & $54(58.7)$ & $46(58.9)$ & $\begin{array}{l}64.40 \\
\pm \\
12.60\end{array}$ & $\begin{array}{l}63.70 \\
\pm \\
11.90\end{array}$ & N/A & $\mathrm{N} / \mathrm{A}$ & $\begin{array}{l}\text { TXA: } 2.0 \mathrm{~g} \text {, intraarticular } \\
\text { injection. } \\
\text { Rivoraxaban: } \\
10 \mathrm{mg} / \text { day, orally. }\end{array}$ & $\begin{array}{l}250 \mathrm{ml} 0.9 \% \\
\text { NS: } \\
\text { intravenous } \\
\text { drip. }\end{array}$ & TK \\
\hline $\begin{array}{l}\text { Wen } \\
\text { Li[29] }\end{array}$ & 2018 & China & 33 & 33 & $13(44.2)$ & $16(48.5)$ & $\begin{array}{l}67.80 \\
\pm 7.20\end{array}$ & $\begin{array}{l}68.50 \\
\pm 7.80\end{array}$ & N/A & N/A & $\begin{array}{l}\text { TXA: } 1.0 \mathrm{~g} \text {, intraarticular } \\
\text { injection. } \\
\text { Rivoraxaban: } \\
10 \text { mg/day, orally. }\end{array}$ & $\begin{array}{l}\text { Rivoraxaban: } \\
10 \mathrm{mg} / \text { day, } \\
\text { orally. }\end{array}$ & TK \\
\hline $\begin{array}{l}\text { Xingjing } \\
\text { Wu[30] }\end{array}$ & 2018 & China & 73 & 73 & $\mathrm{~N} / \mathrm{A}$ & $\mathrm{N} / \mathrm{A}$ & 860 & $\otimes 60$ & N/A & $\mathrm{N} / \mathrm{A}$ & $\begin{array}{l}\text { TXA: } 2.0 \mathrm{~g} \text {, intraarticular } \\
\text { injection. } \\
\text { Rivoraxaban: } \\
10 \mathrm{mg} / \text { day, orally. }\end{array}$ & $\begin{array}{l}\text { Rivoraxaban: } \\
10 \mathrm{mg} / \text { day, } \\
\text { orally. }\end{array}$ & Tr \\
\hline $\begin{array}{l}\text { A. Clavé } \\
\text { a[12] }\end{array}$ & 2019 & France & 76 & 75 & $45(59.2)$ & $42(56.0)$ & 65.00 & 64.40 & 27.5 & 26.6 & $\begin{array}{l}\text { TXA: } 1.0 \mathrm{~g} \text {, intravenous } \\
\text { injection, at } 0 \text { hour and } \\
\text { postoperative hour } 3 . \\
\text { Rivoraxaban: } \\
10 \mathrm{mg} / \text { day, orally. }\end{array}$ & $\begin{array}{l}\text { Rivoraxaban: } \\
10 \mathrm{mg} / \text { day, } \\
\text { orally. }\end{array}$ & Tr \\
\hline $\begin{array}{l}\text { A. Clavé } \\
\text { b[12] }\end{array}$ & 2019 & France & 78 & 75 & $44(56.4)$ & $42(56.0)$ & 67.10 & 64.40 & 25.3 & 26.6 & $\begin{array}{l}\text { TXA: } 1.0 \mathrm{~g} \text {, intravenous } \\
\text { injection, at } 0 \text { hour and } \\
\text { postoperative hours } 3,7 \text {, } \\
\text { and } 11 . \\
\text { Rivoraxaban: } \\
10 \mathrm{mg} / \text { day, orally. }\end{array}$ & $\begin{array}{l}\text { Rivoraxaban: } \\
10 \mathrm{mg} / \text { day, } \\
\text { orally. }\end{array}$ & Tr \\
\hline
\end{tabular}


The Cochrane Risk of Bias criteria was used to evaluate the quality of the retrieved studies by two authors. The included studies were all randomized controlled trials. 16 studies $[12,16-30]$ described random sequence generation and allocation concealment. 4 studies $[12,16,18,24]$ described blinding of participants and personnel. 4 studies $[12,16,18,24]$ described blinding of outcome assessment. None of the studies described other biases. The literature quality score is shown in Table 2.

Table 2

Assessment of Methodological Quality of Included Studies.

\begin{tabular}{|c|c|c|c|c|c|c|c|}
\hline Study & $\begin{array}{l}\text { Random } \\
\text { allocation }\end{array}$ & $\begin{array}{l}\text { Hidden } \\
\text { distribution }\end{array}$ & $\begin{array}{l}\text { Blind } \\
\text { method }\end{array}$ & $\begin{array}{l}\text { Incomplete Outcome } \\
\text { Data }\end{array}$ & $\begin{array}{l}\text { Selective reporting of } \\
\text { results }\end{array}$ & $\begin{array}{l}\text { Other } \\
\text { bias }\end{array}$ & $\begin{array}{l}\text { Quality } \\
\text { grade }\end{array}$ \\
\hline Jianbao Li[16] & Randomized & No clear & $\begin{array}{l}\text { Single- } \\
\text { blind }\end{array}$ & Low & Low & Low & C \\
\hline $\begin{array}{l}\text { Guokuan } \\
\text { Xing[17] }\end{array}$ & Randomized & No clear & No clear & Low & Low & Low & $\mathrm{C}$ \\
\hline $\begin{array}{l}\text { Junwen } \\
\text { Wang[18] }\end{array}$ & Randomized & No clear & $\begin{array}{l}\text { Double- } \\
\text { blind }\end{array}$ & Low & Low & Low & B \\
\hline Fulin Li[19] & Randomized & No clear & No clear & Low & Low & Low & $\mathrm{C}$ \\
\hline $\begin{array}{l}\text { Guokuan } \\
\text { Xing[20] }\end{array}$ & Randomized & No clear & No clear & Low & Low & Low & C \\
\hline Hongjian Xu[21] & Randomized & No clear & No clear & Low & Low & Low & $\mathrm{C}$ \\
\hline Jinwei Xie[22] & Randomized & No clear & No clear & Low & Low & Low & B \\
\hline Keming Xia[23] & Randomized & No clear & No clear & Low & Low & Low & C \\
\hline $\begin{array}{l}\text { ShihHsiang } \\
\text { Yen[24] }\end{array}$ & Randomized & No clear & $\begin{array}{l}\text { Double- } \\
\text { blind }\end{array}$ & Low & Low & Low & B \\
\hline Weina Zhou[25] & Randomized & No clear & No clear & Low & Low & Low & C \\
\hline Yanmei Fan[26] & Randomized & No clear & No clear & Low & Low & Low & C \\
\hline Yanan Fan[27] & Randomized & No clear & No clear & Low & Low & Low & $\mathrm{C}$ \\
\hline Fang Lan[28] & Randomized & No clear & No clear & Low & Low & Low & C \\
\hline Wen Li[29] & Randomized & No clear & No clear & Low & Low & Low & C \\
\hline Xingjing Wu[30] & Randomized & No clear & No clear & Low & Low & Low & C \\
\hline A. Clavé[12] & Randomized & No clear & $\begin{array}{l}\text { Double- } \\
\text { blind }\end{array}$ & Low & Low & Low & B \\
\hline
\end{tabular}

\section{Primary Endpoints}

\section{TBL}

Thirteen studies $[12,16-18,20-24,26,27,29,30]$ (16 trial comparisons) reported TBL. In total, 1712 patients were involved to evaluate TBL, wherein 936 were assigned to experimental group and 776 were assigned to control group. The result showed that patients' TBL in experimental group was significantly less than that in control group (SMD: $-1.34,95 \% \mathrm{Cl}-2.03$ to $-0.64, \mathrm{I}^{2}=97.7 \%$ ) as shown in Fig. 2 . The random effect model was applied. Subgroup analysis was performed according to the administration, operative type, follow-up period and dosage of TXA.

The result of the administration subgroup showed that patients' TBL in experimental group was significantly less than that in control group when TXA were local wet compressed or intravenous injected (SMD: $-2.22,95 \% \mathrm{Cl}-3.21$ to-1.23; SMD: $-0.76,95 \% \mathrm{Cl}-0.99$ to -0.52 ); there was no significant difference when TXA were intravenous dripped, intraarticular injected or intravenous dripped and intraarticular injected at the same time (SMD: $-0.63,95 \% \mathrm{Cl}-1.42$ to 0.16 ; SMD: $-1.87,95 \% \mathrm{Cl}-3.82$ to 0.07 ; SMD: $-1.05,95 \% \mathrm{Cl}-2.60$ to 0.51 ).

The result of operative type subgroup showed that patients' TBL in experimental group was significantly less than that in control group when undergoing THA (SMD: $-2.43,95 \% \mathrm{Cl}-3.84$ to-1.01); there was no significant difference between the TXA group and control group when undergoing TKA (SMD: -0.73 , 95\% Cl -1.53 to 0.06$)$.

The result of the follow-up period subgroup showed that patients' TBL in experimental group was significantly less than that in control group when the followup period was half a month or 6 months (SMD: $-1.63,95 \% \mathrm{Cl}-2.37$ to-0.89; SMD: $-4,85,95 \% \mathrm{Cl}-7.82$ to -1.87 ); there was no significant difference between experimental group and control group when the follow-up period was 3 months (SMD: $-0.15,95 \% \mathrm{Cl}-0.80$ to 0.49 ).

The result of dosage subgroup showed that patients' TBL in experimental group was significantly less than that in control group at $1 \mathrm{~g}$ or $2 \mathrm{~g}$ dosage (SMD: $-1.11,95 \% \mathrm{Cl}-2.10$ to -0.13 ; SMD: $-2.99,95 \% \mathrm{Cl}-4.86$ to -1.12$)$; there was no significant difference between experimental group and control group at other dosage (SMD: $-0.64,95 \% \mathrm{Cl}-1.81$ to 0.53 ). 
Four studies $[16,17,20,21]$ (4 trial comparisons) reported BTV. In total, 460 patients were involved to evaluate BTV, wherein 230 were assigned to the experimental group and 230 were assigned to control group. The result showed that patients' BTV in experimental group was significantly less than that in control group (SMD: $-1.42,95 \% \mathrm{Cl}-2.04$ to $-0.80, \mathrm{I}^{2}=88.8 \%$ ) as shown in Fig. 3 . The random effect model was applied. Subgroup analysis was performed according to the operative type. The result of operative type subgroup showed that patients' BTV in experimental group was significantly less than that in control group when undergoing THA or TKA (SMD: $-1.60,95 \% \mathrm{Cl}-2.25$ to -0.95 ; SMD: $-1.25,95 \% \mathrm{Cl}-2.49$ to- -0.01 )

\section{BTR}

Thirteen studies [16-18, 20-24, 26-30] (16 trial comparisons) reported blood transfusion. In total, 77 out of 950 patients in experimental group experienced blood transfusion while 158 out of 784 patients in the control group experienced blood transfusion. The result showed that TXA combined with rivaroxaban significantly reduce the incidence of blood transfusion compared to the control group ( $8.1 \%$ vs $20.2 \%)\left(\mathrm{RR}: 0.39,95 \% \mathrm{Cl} 0.31\right.$ to $\left.0.49 \mathrm{I}^{2}=0.0 \%\right)$ as shown in Fig. 4. The fixed effect model was applied. Subgroup analysis was performed according to the administration, operative type, follow-up period and dosage of TXA.

The result of administration subgroup showed that TXA combined with rivaroxaban significantly reduce the incidence of blood transfusion compared to the control group when TXA was intravenous driped, intraarticular injected, intravenous driped and intraarticular injected or local wet compressed at the same time (RR: $0.47,95 \% \mathrm{Cl} 0.32$ to 0.68 ; RR: $0.35,95 \% \mathrm{Cl} 0.23$ to 0.53 ; RR: $0.37,95 \% \mathrm{Cl} 0.22$ to 0.63 ; RR: $0.33,95 \% \mathrm{Cl} 0.16$ to 0.72 ).

The result of operative type subgroup showed that TXA combined with rivaroxaban significantly reduce the incidence of blood transfusion compared to the control group when undergoing THA or TKA (RR:0.36, $95 \% \mathrm{Cl} 0.24$ to 0.55 ; RR:0.40, $95 \% \mathrm{Cl} 0.30$ to 0.54 ).

The result of follow-up period subgroup showed that TXA combined with rivaroxaban significantly reduce the incidence of blood transfusion compared to the control group when the follow-up period was half a month, 1 month, 3 months, 6 months (RR:0.39, $95 \% \mathrm{Cl} 0.28$ to 0.55 ; RR:0.33, $95 \% \mathrm{Cl} 0.18$ to 0.61 ; RR:0.37, $95 \% \mathrm{Cl} 0.23$ to 0.59 ; RR:0.52, $95 \% \mathrm{Cl} 0.28$ to 0.99$)$.

The result of dosage subgroup showed that TXA combined with rivaroxaban significantly reduce the incidence of blood transfusion compared to the control group at $1 \mathrm{~g}, 2 \mathrm{~g}$ or other dosage (RR:0.36, $95 \% \mathrm{Cl} 0.19$ to $0.69 ; \mathrm{RR}: 0.41,95 \% \mathrm{Cl} 0.26$ to $0.64 ; \mathrm{RR}: 0.39,95 \% \mathrm{Cl} 0.29$ to 0.53 ).

\section{Secondary Endpoints}

The result showed that compared to the control group, TXA combined Rivaroxaban could significantly reduce the HBL (SMD: $-0.89,95 \% \mathrm{Cl}-1.62$ to $-0.17, \mathrm{I}^{2}=$ 97.5\%); IBL(SMD:-0.64, 95\% Cl -1.15 to $-0.12, \mathrm{I}^{2}=93.7 \%$ ); postoperative drainage (SMD: $-1.37,95 \% \mathrm{Cl}-2.02$ to $-0.72, \mathrm{I}^{2}=96.8 \%$ ); PT (SMD: $-1.01,95 \% \mathrm{Cl}-1.93$ to $\left.-0.09, \mathrm{I}^{2}=97.0 \%\right)$.

The result showed that compared to the control group, TXA combined Rivaroxaban could significantly increase the APTT (SMD: $0.26,95 \%$ CI 0.13 to $0.38, I^{2}=$ $5.6 \%)$; $\mathrm{Hb}$ (SMD: $1.49,95 \% \mathrm{Cl} 0.24$ to $\left.2.74, \mathrm{I}^{2}=97.9 \%\right)$.

There was no significant difference between the TXA combined Rivaroxaban and the control group on FG (SMD: $-0.12,95 \% \mathrm{Cl}-0.32$ to $0.07, \mathrm{I}^{2}=0.0 \%$ ).

\section{Safety Endpoints}

The result showed that compared to the control group, TXA combined Rivaroxaban could significantly reduce the incidence of MB (1.5\% vs $3.4 \%$ ) (RR: 0.27 , $95 \% \mathrm{Cl} 0.10$ to $\left.0.71, \mathrm{I}^{2}=27.5 \%\right)$.

There was no significant difference between the TXA combined Rivaroxaban and the control group on NMB (21.0\% vs $28.1 \%)$ (RR: $0.85,95 \%$ CI 0.69 to $1.04, I^{2}$ $=48.2 \%$ ) and VTE (3.34\% vs $4.35 \%$ ) (RR: $0.80,95 \% \mathrm{Cl} 0.51$ to $1.26, \mathrm{I}^{2}=0.0 \%$ ).

\section{Publication Bias And Sensitivity Analysis}

The funnel plot showed that there was bias among retrieved articles as shown in Supply Fig. 1-7. The results of the sensitivity analysis were shown in Supply Fig. 8-10.

\section{Discussion}

THA/TKA is one of the operations with large blood loss in orthopedic surgery [31]. TXA, a hemostatic, is often used to prevent perioperative bleeding in TKA/THA [32]. However, the antifibrinolytic effect of TXA may increase the risk of DVT [33]. Anticoagulant drugs should be given within 6-12 hours after the application of TXA [34]. As a direct oral anticoagulant, rivaroxaban has been used clinically for more than a decade, and its antithrombotic effect has been widely recognized [35]. Applying TXA and rivaroxaban at the same time in clinic is contradictory, so the efficacy and safety of TXA combined with rivaroxaban in the prevention of clinical events in patients undergoing TKA/THA are still controversial [36]. 
Nowadays, there are many meta-analyses to study TXA in patients after THA/TKA. They all concluded that TXA was effective after THA/TKA. Grandhi et al [37], Wei et al [14], Dong et al [38], and Kuo et al [39] conducted meta-analyses to evaluate the effectiveness and safety of aminocaproic acid for reducing blood loss in total knee and hip arthroplasty; Li et al [40], Chen et al [41] and Yang et al [42] conducted meta-analyses to comprise the efficacy and safety of topical, system and intravenous tranexamic acid usage in total knee and hip arthroplasty; Zhang et al [43] and Han et al [44] conducted meta-analyses to compared the efficacy and safety of oral compared with intravenous tranexamic acid in reducing blood loss after primary total knee and hip arthroplasty. However, they only focused on the administration of TXA itself, but did not analysed other influencing factors. Yu et al [45] and Wu et al [46] compared tranexamic acid plus diluted-epinephrine versus tranexamic acid alone for blood loss in total joint arthroplasty, but they only retrieved several studies and only focused on blood loss and transfusion rate. As a result, whether TXA combined with anticoagulant are effective and safe enough to apply in clinical is still inconsistent.

This is the first meta-analysis to evaluate the efficacy and safety of TXA combined with rivaroxaban in the prevention of clinical events in patients undergoing TKA/THA. The result showed that TXA combined with rivaroxaban significantly reduce TBL, BTV, BTR and the incidence of MB compared to the control group (SMD: $-1.34,95 \% \mathrm{Cl}-2.03$ to -0.64 ; SMD: $-1.42,95 \% \mathrm{Cl}-2.04$ to -0.80 ; RR: $0.39,95 \% \mathrm{Cl} 0.31$ to 0.49 ; RR: $0.27,95 \% \mathrm{Cl} 0.10$ to 0.71 ); there were no significant differences in NMB between experimental group and control group (RR: $0.85,95 \% \mathrm{Cl} 0.69$ to 1.04 ).

When evaluating the primary endpoints, we found that the results were highly heterogeneous, so we did sensitivity analyses to decompose it. The results showed that after excluding Wu et al 's article [30], the overall effect of TBL has been greatly affected; after excluding Wang et al 's article [18], the overall effect of NMB has been greatly affected; the heterogeneity of BTV cannot be explore by sensitivity analysis, which may be caused by the lack of studies. In the study of Wu et al [46], the TBL was calculated according to the formula provided by Good et al [47], which was somewhat different from the calculation methods in other studies. (In this formula, the TBL was calculated without blood transfusion.) It would easily lead to heterogeneity; in the study of Wang et $\mathrm{al}^{14}{ }^{14}$ rivaroxaban was used in control group. As a direct oral anticoagulant, it is not surprising that the use of rivaroxaban increases the risk of NMB.

Subgroup analyses were performed according to the administration, operative type, follow-up period and dosage of TXA when evaluating primary endpoints. And subgroup analyses were performed only if there were more than two trial comparisons per subgroup. In the administration that TXA combined with rivaroxaban was more effective in reducing TBL during local wet compression or intravenous injection Thus, the administration should be paid attention to in the clinical use. In the operative type subgroup analysis, the effects of TXA combined with rivaroxaban on patients undergoing THA or TKA were different, which may be due to the different wound size, operation time and the severity of primary disease. In the follow-up time subgroup analysis, the results showed that except that the 3-month group had no effect on TBL, each group could reduce TBL and BTR. This may be due to the imbalance in the number of people in each subgroup. In the dosage of TXA subgroup analysis, the results showed that except that other group had no effect on TBL, each group could reduce TBL and BTR. Because the dosage of TXA included in the article is different, we only distinguish it from the clinical commonly used $1 \mathrm{~g}$ and $2 \mathrm{~g}$, and combine the other doses into one group, so different doses may be the main reason for the invalidity of other group.

The potential clinical implications of this meta-analysis are as follows: (1) This is the first study focusing on the efficacy and safety of TXA combined with rivaroxaban in patients after THA/TKA. Previous articles evaluated the efficacy of TXA after THA/TKA, but there was no specific meta-analysis to assess the applying of TXA combined with rivaroxaban. Our article just filled the gap. (2) 16 RCTs were retrieved which included a large sample size of 2179 participants compared to previous studies. (3) Subgroup analyses were performed according to the administration, operative type, follow-up period and dosage of TXA to explain the influence of different factors on the overall effect. (4) Sensitivity analyses were conducted to decompose heterogeneity and explore the influence of sample size on the overall effect. (5) We evaluated 12 indicators, including TBL, BTV, BTR, HBL, IBL, postoperative drainage, APTT, FG, Hb, PT, MB and NMB, which were more comprehensive than previous articles.

The limitations of this study are as follows: (1) Several baseline characteristics (diabetes, hypertension, older age or other drug use) were not considered and this may lead to mixed bias. (2) We used the outcome events reported in the retrieved studies to integrate the results of this meta-analysis. Therefore, it is difficult to assess the effect of these baseline characteristics on the results. (3) This study could not explore the interactions among the subgroup analysis because of the limitations inherent in the included studies. (4) The intervention measures in the control group were different. Some groups were given saline intravenously, some groups were treated with rivaroxaban or TXA alone and the dosage was different, so subgroup analysis could not be carried out, which may lead to significant heterogeneity. However, in view of ethical factors, it is immoral to require the original author not to use any hemostatic or anticoagulant interventions, so we have included all of these articles. (5) Only two retrieved articles have been published in what are considered high-impact orthopaedic surgical journals of the English literature. Seven of the retrieved papers have been published in Chinese journals, and the remaining in journals of general medicine. As a result, we have correctly tried to address this issue by evaluating the quality of the retrieved studies, assigning a grade $\mathrm{C}$ to most.

\section{Conclusion}

This meta-analysis reveals that TXA combined with rivaroxaban can significantly reduce TBL, BTV, the incidence of blood transfusion and the incidence of MB compared to the control group, which proved that its efficacy and safety are trustworthy.

\section{Abbreviations}

$\mathrm{RCT}=$ Randomized Controlled Trials, $\mathrm{RR}=$ Risk ratios, $\mathrm{SMD}=$ Standard mean difference $\mathrm{Cl}=$ confidence intervals, $\mathrm{TXA}=\mathrm{Tranexamic}$ acid, $\mathrm{TBL}=$ total blood loss, $\mathrm{BTV}=$ blood transfusion volume, $\mathrm{BTR}=$ blood transfusion rate, $\mathrm{HBL}=$ hidden blood loss, IBL = intraoperative blood loss, $\mathrm{APTT}=$ activated partial thromboplastin time, $\mathrm{FG}=$ fibrinogen, $\mathrm{Hb}=$ hemoglobin, $\mathrm{PT}=$ prothrombin time. $\mathrm{MB}=$ major bleeding, $\mathrm{NMB}=$ non-major bleeding, $\mathrm{VTE}=\mathrm{venous}$ thromboembolism. 


\section{Declarations}

\section{Ethics approval and consent to participate}

Not applicable.

\section{Consent for publication}

Not applicable.

\section{Availability of data and materials}

Not applicable.

\section{Competing interests}

The authors declare that they have no competing interests.

\section{Funding}

None.

\section{Authors' contributions}

M.J. design the study; M.J. and H.D. pooled the data; M.J. analysed the data; M.J., S.L. and X.X wrote the article; B.Z. reviewed the article. All Authors read and approved the manuscript

\section{Acknowledgements}

This study was supported by Guangxi Medical University First Affiliated Hospital.

\section{References}

1. Xenakis TA, Gelalis J, Koukoubis TA, Zaharis KC, Soucacos PN. Cementless hip arthroplasty in the treatment of patients with femoral head necrosis. Clin Orthop Relat Res. 2001; 386(386):93-99.

2. Levy O, Martinowitz U, Oran A, Tauber C, Horoszowski $\mathrm{H}$. The use of fibrin tissue adhesive to reduce blood loss and the need for blood transfusion after total knee arthroplasty. A prospective, randomized, multicenter study. Journal of Bone \& Joint Surgery American Volume. 1999;81(11):1580-1588.

3. Hölscher AH, Vallböhmer D, Brabender J. The prevention and management of perioperative complications. Best Practice \& Research Clinical Gastroenterology.2006;20(5):907-923.

4. Demos HA, Lin ZX, Barfield WR, Wilson SH, Robertson DC, Jr VDP. Process Improvement Project Using Tranexamic Acid Is Cost-Effective in Reducing Blood Loss and Transfusions After Total Hip and Total Knee Arthroplasty. J Arthroplasty. 2017:S0883540317301882.

5. Aydın B, Durgut F, Erkoçak Ö, Acar MA. Other benefits of intra-articular injection of tranexamic acid in primary total knee arthroplasty apart from reducing blood transfusion rates. Eklem hastaliklari ve cerrahisi= Joint diseases \& related surgery. 2017;28(1):25-29.

6. Wu Y, Yang T, Zeng Y, Li C, Shen B, Pei F. Clamping drainage is unnecessary after minimally invasive total knee arthroplasty in patients with tranexamic acid: A randomized, controlled trial. Medicine. 2017;96(7):e5804.

7. Jacobs JJ, Mont MA, Bozic KJ, Della Valle CJ, Goodman SB, Lewis CG, Jr YA, Boggio LN, Rd WW, Turkelson CM. American Academy of Orthopaedic Surgeons clinical practice guideline on: preventing venous thromboembolic disease in patients undergoing elective hip and knee arthroplasty. Journal of Bone \& Joint Surgery American Volume. 2012;94(8):746-747.

8. Falck-Ytter Y, Francis CW, Johanson NA, Curley C, Dahl OE, Schulman S, Ortel TL, Pauker SG, Jr CWC. Prevention of VTE in Orthopedic Surgery Patients : Antithrombotic Therapy and Prevention of Thrombosis, 9th ed: American College of Chest Physicians Evidence-Based Clinical Practice Guidelines. Chest. 2012;141(2):e278S.

9. Myers B, Neal R, Myers O, Ruparelia M. Unplanned pregnancy on a direct oral anticoagulant (Rivaroxaban): A warning. Obstetric Medicine. 2016;9(1):40.

10. Jameson SS, Monika R, Hui ACW, Philip J, Ignacio SP, Muller SD. Wound complications following rivaroxaban administration: a multicenter comparison with low-molecular-weight heparins for thromboprophylaxis in lower limb arthroplasty. Journal of Bone \& Joint Surgery American Volume. 2012;94(17):1554-1558.

11. Melvin JS, Stryker LS, Sierra RJ. Tranexamic Acid in Hip and Knee Arthroplasty. J Am Acad Orthop Surg.2015; 23(12):732-740.

12. Clavé A, Gérard R, Lacroix J, Baynat C, Danguy dD, M, Gatineau F, Mottier D. A randomized, double-blind, placebo-controlled trial on the efficacy of tranexamic acid combined with rivaroxaban thromboprophylaxis in reducing blood loss after primary cementless total hip arthroplasty. The Bone \& Joint Journal. 2019;null(2):207-212.

13. Gillette BP, Desimone LJ, Trousdale RT, Pagnano MW, Sierra RJ. Low Risk of Thromboembolic Complications With Tranexamic Acid After Primary Total Hip and Knee Arthroplasty. Clinical Orthopaedics \& Related Research. 2012;471(1):150-154.

14. Wei Z, Liu M. The effectiveness and safety of tranexamic acid in total hip or knee arthroplasty: a meta-analysis of 2720 cases. Transfus Med. 2015;25(3):151-162. 
15. Sabbag OD, Abdel MP, Amundson AW, Larson DR, Pagnano MW. Tranexamic Acid Was Safe in Arthroplasty Patients With a History of Venous Thromboembolism: A Matched Outcome Study. J Arthroplasty. 2017;32(9S):S246.

16. Li JB. The effects of tranexamic acid on the perioperative blood loss in the primary unilateral cementless total hip replacement. Fujian medical university. 2014.

17. Xing GK, Liu XF, Fan JQ, Chen G. Tranexamic acid combined with rivaroxaban in reducing bleeding and prevention of venous thrombosis in knee arthroplasty. Practical Pharmacy And Clinical Remedies. 2016;19(3):326-330.

18. Wang JW, Chen B, Lin PC, Yen SH, Huang CC, Kuo FC. The Efficacy of Combined Use of Rivaroxaban and Tranexamic Acid on Blood Conservation in Minimally Invasive Total Knee Arthroplasty a Double-Blind Randomized, Controlled Trial. J Arthroplasty.2017;32(3):801-806.

19. Li FL, Yin D, Mo BF, Huang Y, Huang X, Lu Q, Liu WH. Study on efficacy and safety of sequential rivaroxaban use in reducing blood loss after applying tranexamic acid in total hip arthroplasty. Chongqing Med. 2017;46(16):2193-2197.

20. Xing GK, Liu XF, Fan JQ, Chen G. Clinical Efficacy and Safety of Tranexamic Acid Combined with Rivaroxaban in Preventing Perioperative Compli-cations in Patients Receiving Total Knee Arthroplasty. Medical Recapitulate.2017; 23(17):3511-3514

21. Xu HJ, Zhong YP, Wu CM. Effect of rivaroxaban combined with tranexamic acid on the hemorrhage and transfusion in hip replacement surgery. Clinical Education of General Practice. 2017; 15(4): 412-414.

22. Xie J, Ma J, Huang Q, Yue C, Pei F. Comparison of Enoxaparin and Rivaroxaban in Balance of Anti-Fibrinolysis and Anticoagulation Following Primary Total Knee Replacement: A Pilot Study. Med Sci Monit. 2017;23:704-711.

23. Xia KM, Zhu WM, Yang B, Yang P. Effect of Tranexamic Acid injection on blood loss during the perioperative period of total knee arthroplasty. China Journal of Modern Med. 2017; 27(24):101-104.

24. Yen SH, Lin PC, Chen B, Huang CC, Wang JW. Topical Tranexamic Acid Reduces Blood Loss in Minimally Invasive Total Knee Arthroplasty Receiving Rivaroxaban. Biomed Res Int. 2017:9105645.

25. Zhou WN, Zhang Y, Cao XY, Gu R. [Observation on the effect of oral rivaroxaban in the prevention of deep venous thrombosis of lower extremities after unilateral total hip replacement.] (In Chinese). Shandong Med. 2017;57(44):95-97.

26. Fan YM, Yang JX, Zhao J, Guo HL, Wu HY. Effect of tranexamic acid combined with rivaroxaban on blood loss and safety in elderly patients with total knee replacement. J Trop Med. 2017;17(01):69-71+94.

27. Fan YN, Wen YY, Li WL, Zhu YJ, Liu YW. Analysis of the application of Rivaroxaban after anti -fibrinolysis with Tranexamic acid in total hip arthroplasty. China Medical Herald. 2017;14(36).

28. Lan F, Lin WL, Lin Y. [Effect of intravenous infusion of tranexamic acid on occult bleeding after the first unilateral total knee replacement.] (In Chinese). Modern Practical Medicine. 2018; 30: 910-912.

29. Li W, Yang XH, Zeng GQ, Zhou YW, Gao CY. Effect of Tranexamic Acid Combined Rivaroxaban in Bleeding Volume, Coagulation Function, Knee Function in Patients with Unilateral Total Knee Arthroplasty. Progress in Modern Biomedicine. 2018;18(09):140-144.

30. Wu XJ, Wang KZ, Fan LH, et al. Clinical efficacy of rivaroxaban combined with topical application of tranexamic acid in primary total hip arthroplasty. Chin J Joint Surg. 2018;12(3):300.

31. Hao W, Bin S, Yi Z. Comparison of topical versus intravenous tranexamic acid in primary total knee arthroplasty: a meta-analysis of randomized controlled and prospective cohort trials. Knee. 2014;21(6):987-993.

32. Winter SF, Santaguida C, Wong J, Fehlings MG. Systemic and Topical Use of Tranexamic Acid in Spinal Surgery: A Systematic Review. Global Spine Journal. 2015;06(03):284-295.

33. Huang F, Wu Y, Yin Z, Ma G, Chang J. A systematic review and meta-analysis of the use of antifibrinolytic agents in total hip arthroplasty. Hip Int. 2015;25(6):502-509.

34. Tzong-Huei C, Robina M. The management of antiplatelet therapy in patients with coronary stents undergoing noncardiac surgery. Semin Cardiothorac Vasc Anesth. 2010;14(4):256-273.

35. Helen M, Natalie H, Alexander K, Thomas W, Gundolf S, Yvonne W, Waltraud P, Birgit L, Eva H, Edelgard LL. Point-of-care coagulation testing for assessment of the pharmacodynamic anticoagulant effect of direct oral anticoagulant. Ther Drug Monit. 2014;36(5):624-631.

36. Jan BW, J?Rg L, Lars D, Luise T, Holger K, Radke OC, Eberhard K, Thoralf S, Albrecht H, Klaus-Peter G. Efficacy and safety of thromboprophylaxis with lowmolecular-weight heparin or rivaroxaban in hip and knee replacement surgery: findings from the ORTHO-TEP registry. Thromb Haemost. 2013;109(01):154-163.

37. Gandhi R. Tranexamic acid and the reduction of blood loss in total knee and hip arthroplasty: a meta-analysis. BMC Research. 2013;6(1):184-184.

38. Dong Q, Zhang Y, Sun X, Hu F. The effectiveness and safety of aminocaproic acid for reducing blood loss in total knee and hip arthroplasty: A metaanalysis. International Journal of Surgery. 2018;52:156-163.

39. Kuo F-C, Lin P-Y, Wang J-W, Lin P-C, Lee MS, Chen AF. Intravenous tranexamic acid use in revision total joint arthroplasty: a meta-analysis. Drug Des Devel Ther. 2018;12(3163).

40. Li J, Zhang Z, Chen J. Comparison of efficacy and safety of topical versus intravenous tranexamic acid in total hip arthroplasty: A meta-analysis. Medicine. 2016;95(36):e4689.

41. Chen Y, Chen Z, Cui S, Li Z, Yuan Z. Topical versus systemic tranexamic acid after total knee and hip arthroplasty: A meta-analysis of randomized controlled trials. Medicine. 2016;95(41):e4656.

42. Yang L, Du S, Sun Y. Is combined topical and intravenous tranexamic acid superior to single use of tranexamic acid in total joint arthroplasty? Medicine. 2017.

Page $10 / 17$ 
43. Zhang LK, Ma JX, Kuang MJ, Zhao J, Wang Y, Lu B, Sun L, Ma XL. Comparison of oral versus intravenous application of tranexamic acid in total knee and hip arthroplasty: A systematic review and meta-analysis. International Journal of Surgery. 2017;45:77-84.

44. Han X, Gong G, Han N, Liu M. Efficacy and safety of oral compared with intravenous tranexamic acid in reducing blood loss after primary total knee and hip arthroplasty: a meta-analysis. BMC Musculoskelet Disord. 2018;19(1):430.

45. Yu Z, Yao L, Yang Q. Tranexamic acid plus diluted-epinephrine versus tranexamic acid alone for blood loss in total joint arthroplasty: A meta-analysis. Medicine. 2017; 96(24):e7095.

46. Wu YG, Zeng Y, Hu QS, Bao XC, Xiong HZ, Shen B. Tranexamic Acid Plus Low-dose Epinephrine Reduces Blood Loss in Total Knee Arthroplasty: A Systematic Review and Meta-analysis. Orthop Surg. 2018;10.

47. Good L, Peterson E, Lisander B. Tranexamic acid decreases external blood loss but not hidden blood loss in total knee replacement. Br J Anaesth. 2003;90(5):596.

\section{Supplementary Figure Legends}

Supply Figure 1. Comparison of TBL between the experimental group and the control group. (funnel plot)

$\mathrm{SMD}=$ standardized mean difference

Supply Figure 2. Comparison of BTR between the experimental group and the control group. (funnel plot)

$\mathrm{RR}=$ Risk Ratio

Supply Figure 3. Comparison of HBL between the experimental group and the control group. (funnel plot)

$\mathrm{SMD}=$ standardized mean difference

Supply Figure 4. Comparison of IBL between the experimental group and the control group. (funnel plot)

$\mathrm{SMD}=$ standardized mean difference

Supply Figure 5. Comparison of Postoperative drainage between the experimental group and the control group. (funnel plot)

$\mathrm{SMD}=$ standardized mean difference

Supply Figure 6. Comparison of non-major bleeding between the experimental group and the control group. (funnel plot)

$\mathrm{RR}=$ Risk Ratio

Supply Figure 7. Comparison of APTT between the experimental group and the control group. (funnel plot)

$\mathrm{SMD}=$ standardized mean difference

Supply Figure 8. Comparison of TBL between the experimental group and the control group. (sensitivity analysis)

$\mathrm{SMD}=$ standardized mean difference

Supply Figure 9. Comparison of BTV between the experimental group and the control group. (sensitivity analysis)

$\mathrm{SMD}=$ standardized mean difference

Supply Figure 10. Comparison of NMB between the experimental group and the control group. (sensitivity analysis)

$\mathrm{RR}=$ Risk Ratio

\section{Figures}




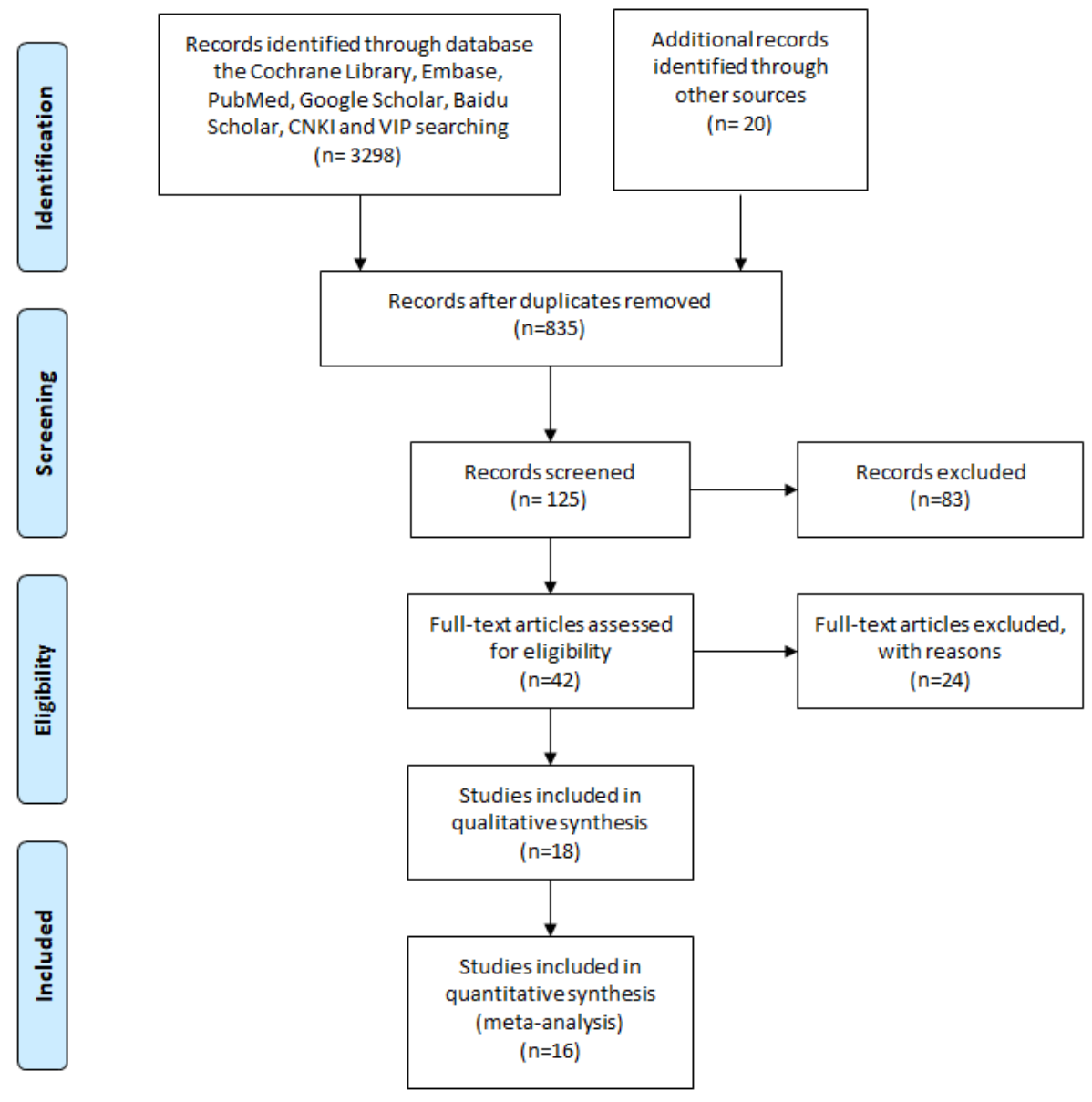

Figure 1

Flow diagram of the study selection process. CNKI=China national knowledge infrastructure, VIP=China Science and Technology Journal Database 


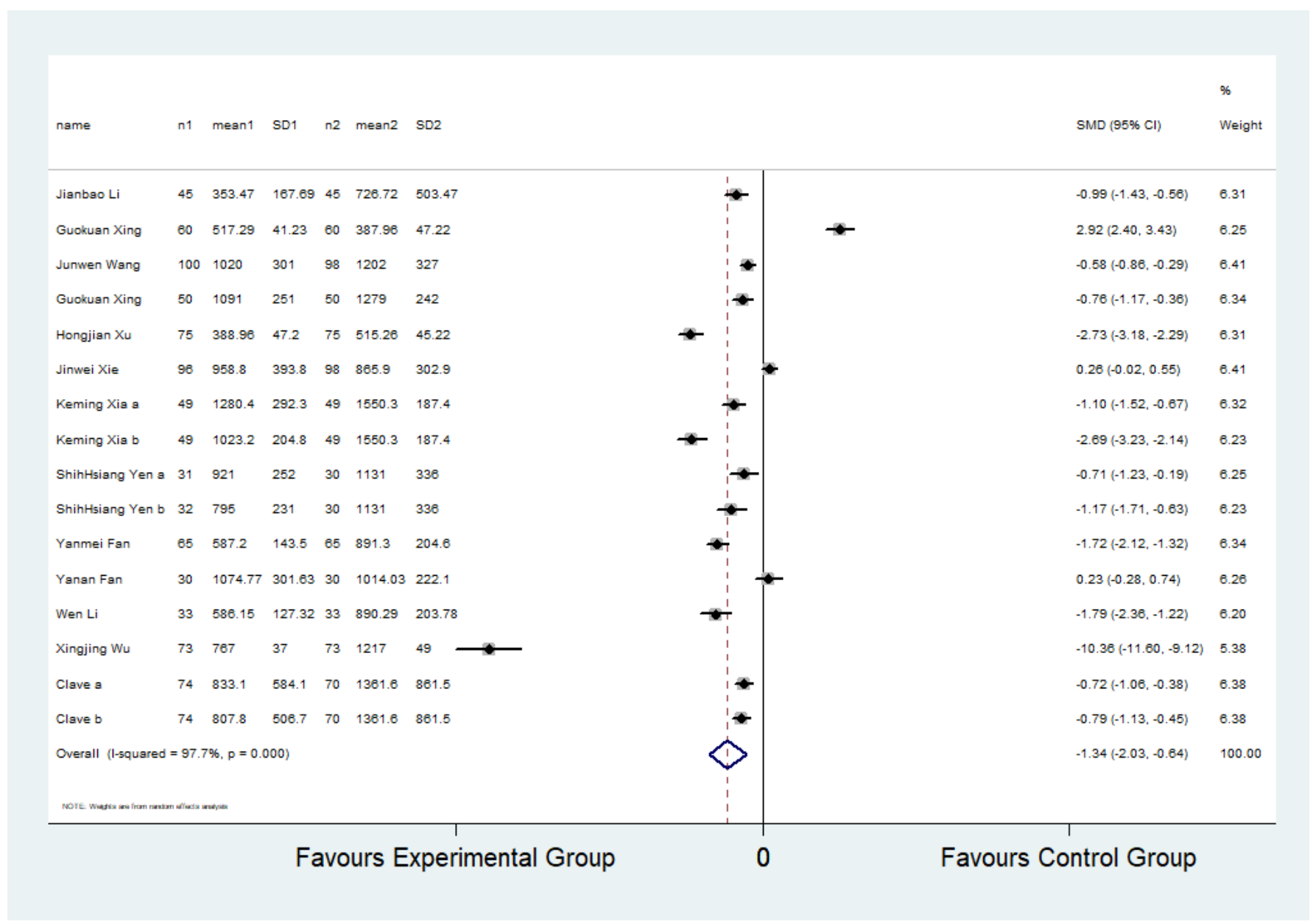

\section{Figure 2}

Comparison of TBL between the experimental group and the control group. SMD= standardized mean difference 


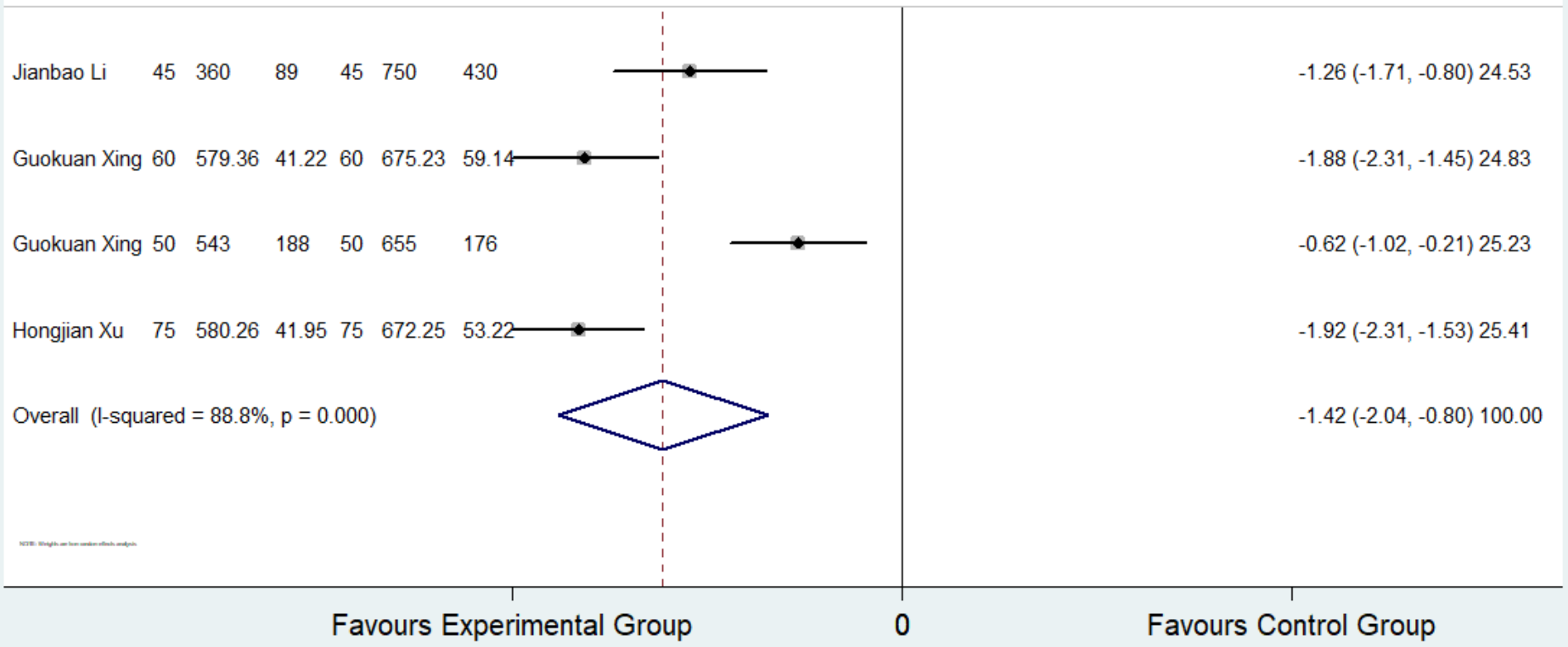

Figure 3

Comparison of BTV between the experimental group and the control group. SMD= standardized mean difference 


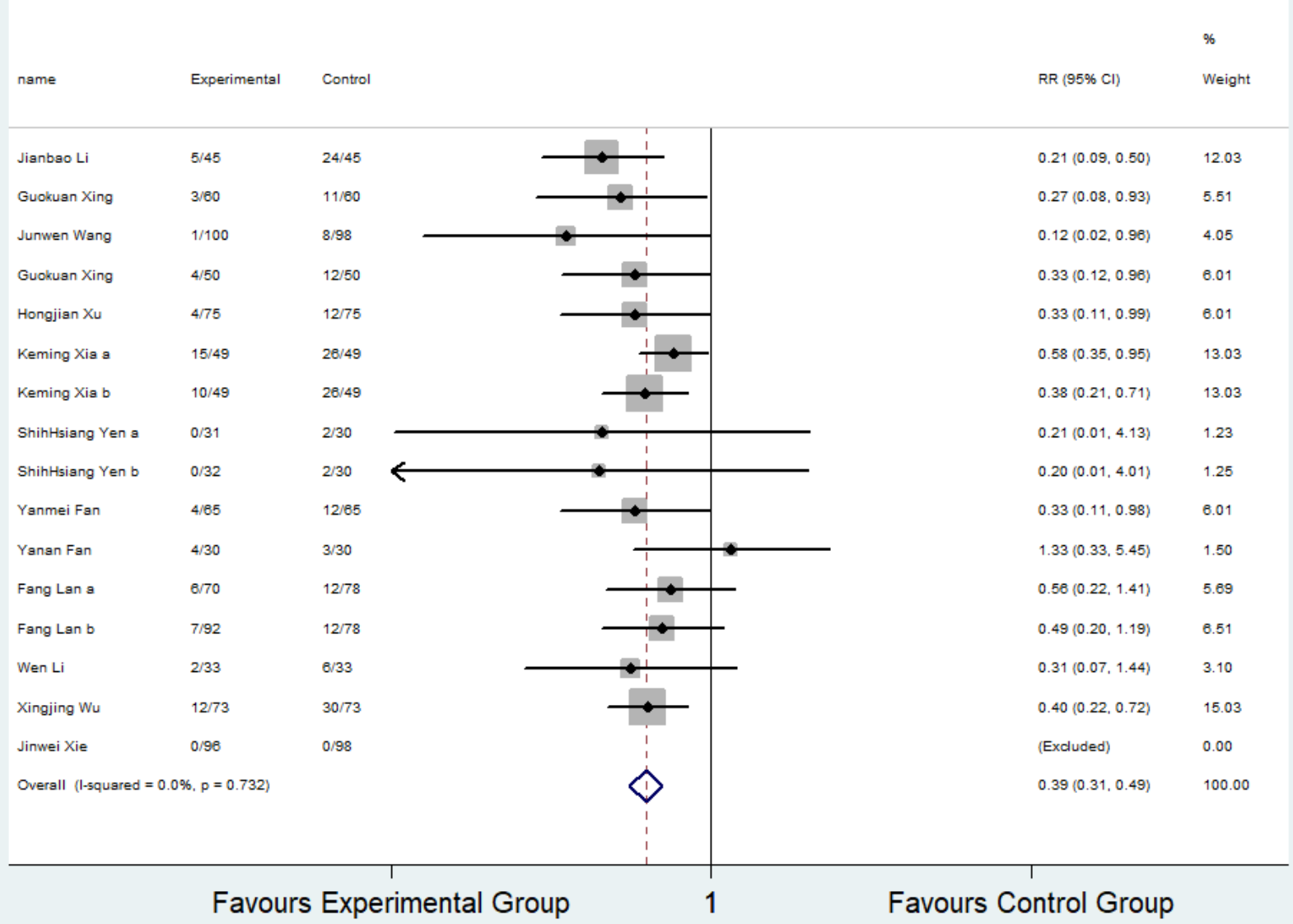

Figure 4

Comparison of BTR between the experimental group and the control group. RR= Risk Ratio 


\begin{tabular}{|c|c|c|c|c|c|}
\hline Primary endpoints & No. of Trials & No. of Patients & $\operatorname{SMD}(95 \% \mathrm{Cl})$ & SMD/RR(95\%Cl) & 12 \\
\hline TBL & 13 & $936 / 776$ & $\because$ & $-1.34(-2.03,-0.64)$ & 97.7 \\
\hline \multicolumn{6}{|l|}{ administration } \\
\hline IV drip & 3 & $124 / 124$ & $\mapsto$ & $-0.63(-1.42,0.16)$ & 88.9 \\
\hline intraarticular injection & 5 & $329 / 324$ & $\longmapsto$ & $-1.87(-3.82,0.07)$ & 98.9 \\
\hline IV drip/intraarticular injection & 3 & $195 / 197$ & $\longmapsto$ & $-1.05(-2.60,0.51)$ & 97.8 \\
\hline local wet compressed & 2 & $140 / 140$ & $\longmapsto$ & $-2.22(-3.21,-1.23)$ & 90.8 \\
\hline IV injection & 1 & $148 / 140$ & - & $-0.76(-0.99,-0.52)$ & 0 \\
\hline \multicolumn{6}{|l|}{ type of operation } \\
\hline THA & 5 & $371 / 363$ & $\longmapsto$ & $-2.43(-3.84,-1.01)$ & 98.3 \\
\hline TKA & 8 & $565 / 562$ & $\mapsto$ & $-0.73(-1.53,0.06)$ & 97.2 \\
\hline \multicolumn{6}{|l|}{ follow-up period } \\
\hline half a month & 3 & $176 / 176$ & $\mapsto-1$ & $-1.63(-2.37,-0.89)$ & 89.1 \\
\hline 3 months & 7 & $547 / 536$ & & $-0.15(-0.80,0.49)$ & 96.1 \\
\hline 6 months & 3 & $213 / 213$ & $=$ & $-4.85(-7.82,-1.87)$ & 98.8 \\
\hline \multicolumn{6}{|l|}{ TXA dosage } \\
\hline $1 \mathrm{~g}$ & 5 & $269 / 266$ & $\longmapsto$ & $-1.11(-2.10,-0.13)$ & 95.9 \\
\hline $2 g$ & 3 & $271 / 263$ & $\longmapsto$ & $-2.99(-4.86,-1.12)$ & 98.7 \\
\hline other & 6 & $396 / 396$ & $\longmapsto$ & $-0.64(-1.81,0.53)$ & 98.1 \\
\hline BTV & 4 & $230 / 230$ & $\mapsto-1$ & $-1.42(-2.04,-0.80)$ & 88.8 \\
\hline \multicolumn{6}{|l|}{ type of operation } \\
\hline THA & 2 & $120 / 120$ & $1-1$ & $-1.60(-2.25,-0.95)$ & 79 \\
\hline \multirow[t]{3}{*}{ TKA } & 2 & $110 / 110$ & $\longmapsto$ & $-1.25(-2.49,-0.01)$ & 94.4 \\
\hline & \multirow{2}{*}{\multicolumn{5}{|c|}{${ }^{-6.00} \mathrm{RR}(95 \% \mathrm{Cl})$}} \\
\hline & & & & & \\
\hline BTR & 13 & $950 / 784$ & $\because$ & $0.39(0.31,0.49)$ & 0 \\
\hline \multicolumn{6}{|l|}{ administration } \\
\hline IV drip & 4 & $194 / 202$ & $\mapsto-1$ & $0.47(0.32,0.68)$ & 51.9 \\
\hline intraarticular injection & 6 & $421 / 372$ & $\mapsto-1$ & $0.35(0.23,0.53)$ & 0 \\
\hline IV drip/intraarticular injection & 3 & $195 / 197$ & $\longmapsto$ & $0.37(0.22,0.63)$ & 0 \\
\hline local wet compressed & 2 & $140 / 140$ & $\mapsto$ & $0.33(0.16,0.72)$ & 0 \\
\hline \multicolumn{6}{|l|}{ type of operation } \\
\hline THA & 4 & $223 / 223$ & $\mapsto-1$ & $0.36(0.24,0.55)$ & 39.6 \\
\hline TKA & 9 & $727 / 561$ & $\mapsto-1$ & $0.40(0.30,0.54)$ & 0 \\
\hline \multicolumn{6}{|l|}{ follow-up period } \\
\hline half a month & 3 & $176 / 127$ & $\mapsto-1$ & $0.39(0.28,0.55)$ & 33 \\
\hline 1 month & 1 & $162 / 78$ & $\mapsto$ & $0.33(0.18,0.61)$ & 0 \\
\hline 3 months & 6 & $399 / 366$ & $\mapsto$ & $0.37(0.23,0.59)$ & 0 \\
\hline 6 months & 3 & $213 / 213$ & $\longmapsto$ & $0.52(0.28,0.99)$ & 0 \\
\hline \multicolumn{6}{|l|}{ TXA dosage } \\
\hline $1 \mathrm{~g}$ & 5 & $269 / 266$ & $\longmapsto$ & $0.36(0.19,0.69)$ & 12.1 \\
\hline $2 g$ & 3 & $215 / 201$ & $\mapsto$ & $0.41(0.26,0.64)$ & 0 \\
\hline \multirow[t]{2}{*}{ other } & 7 & $466 / 425$ & $1-1$ & $0.39(0.29,0.53)$ & 0 \\
\hline & \multicolumn{5}{|c|}{$0.00 \quad 0.50$} \\
\hline
\end{tabular}

Figure 5

Comparison of TBL and BTR between the experimental group and the control group. (subgroup analysis) SMD= standardized mean difference RR= Risk Ratio

\begin{tabular}{|c|c|c|c|c|c|}
\hline Secondary endpoints & No. of Trials & $\begin{array}{c}\text { No. of } \\
\text { Patients }\end{array}$ & $\operatorname{SMD}(95 \% \mathrm{Cl})$ & $\operatorname{SMD}(95 \% \mathrm{Cl})$ & 12 \\
\hline $\mathrm{HBL}$ & 10 & $752 / 619$ & 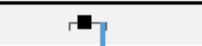 & $-0.89(-1.62,0.17)$ & 97.5 \\
\hline IBL & 7 & $526 / 393$ & 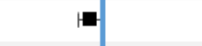 & $-0.64(-1.15,-0.12)$ & 93.7 \\
\hline Postoperative drainage & 10 & $812 / 646$ & -1 & $-1.37(-2.02,-0.72)$ & 96.8 \\
\hline APTT & 8 & $492 / 404$ & $\mathbf{r}$ & $0.26(0.13,0.38)$ & 5.6 \\
\hline FG & 4 & $201 / 201$ & 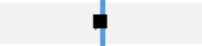 & $-0.12(-0.32,0.07)$ & 0.0 \\
\hline $\mathrm{Hb}$ & 5 & $326 / 277$ & $\mapsto-1$ & $1.49(0.24,2.74)$ & 97.9 \\
\hline \multirow[t]{2}{*}{ PT } & 6 & $397 / 309$ & $1-$ & $-1.01(-1.93,-0.09)$ & 97.0 \\
\hline & & & 0.00 & & \\
\hline
\end{tabular}

\section{Figure 6}

Comparison of $\mathrm{HBL}$, IBL, postoperative drainage, APTT, FG, Hb and PT between the experimental group and the control group. SMD= standardized mean difference 


\begin{tabular}{|c|c|c|c|c|c|}
\hline Safety endpoints & No. of Trials & $\begin{array}{c}\text { No. of } \\
\text { Patients }\end{array}$ & $\mathrm{RR}(95 \% \mathrm{Cl})$ & $\mathrm{RR}(95 \% \mathrm{Cl})$ & 12 \\
\hline MB & 4 & $340 / 263$ & $+\square$ & $0.27(0.10,0.71)$ & 27.5 \\
\hline NMB & 7 & $553 / 441$ & 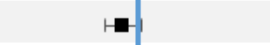 & $0.85(0.69,1.04)$ & 48.2 \\
\hline \multicolumn{6}{|l|}{ TXA dosage } \\
\hline $1 \mathrm{~g}$ & 3 & $206 / 203$ & $\Leftrightarrow-1$ & $0.57(0.40,0.81)$ & 46.2 \\
\hline $2 \mathrm{~g}$ & 2 & $204 / 200$ & $\longmapsto$ & $0.96(0.62,1.48)$ & 0.0 \\
\hline other & 3 & $188 / 188$ & $\mapsto-1$ & $1.11(0.81,1.52)$ & 29.3 \\
\hline \multicolumn{6}{|l|}{ type of operation } \\
\hline THA & 2 & $229 / 225$ & $\longmapsto$ & $0.61(0.25,1.53)$ & 0.0 \\
\hline TKA & 5 & $369 / 366$ & 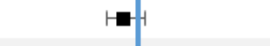 & $0.86(0.70,1.07)$ & 64.8 \\
\hline \multirow[t]{2}{*}{ VTE } & 12 & $897 / 690$ & $\mapsto-1$ & $0.80(0.51,1.26)$ & 0.0 \\
\hline & & & 1.00 & & \\
\hline
\end{tabular}

Figure 7

Incidence of adverse reaction between the experimental group and the control group. RR= Risk Ratio

\section{Supplementary Files}

This is a list of supplementary files associated with this preprint. Click to download.

- 9PRISMADTAChecklist.doc

- SupplyFig.17.tif

- SupplyFig.810.tif 